\title{
Bar-driven injection of intergalactic matter into galactic halos
}

\author{
M. López-Corredoira
}

Instituto de Astrofísica de Canarias, C/Vía Láctea, s/n, 38200 La Laguna (S/C de Tenerife), Spain

e-mail: martinlc@iac.es

Received 8 November 2006 / Accepted 15 March 2007

\section{ABSTRACT}

\begin{abstract}
Aims. The non-conservative gravitational potential of barred galaxies, or of any other non-axisymmetric structure, produces a loss of energy in infalling particles of the intergalactic medium into the galaxy, which are trapped in its potential. This dynamical friction can contribute towards increasing the total mass of barred galaxies.

Methods. Analytical calculations of the energy loss are carried out using the orbits of the particles derived numerically. Theoretical predictions are compared with observations through the statistical analysis of the rotation curves of barred and non-barred galaxies, either in cluster or field galaxies.

Results. There is a net effect of accretion, but it is normally very low in relative terms. It is only significant ( $>10 \%$ of the total mass of the dark matter halo in the life of the galaxy) if the density of the intergalactic medium is higher than $\sim 3 \times 10^{13} M_{\odot} / \mathrm{Mpc}^{3}$ (or considerably lower in cases of motions of the galaxies close to the IGM average motion, or perpendicular to the plane of the galaxy, or when the halo mass is low).

Data on rotation curves do not show clear trends towards higher halo mass for barred galaxies, only slight trends for early-type spiral galaxies. In any case, the statistical uncertainties are limited to the detection of differences in masses $>\approx 20 \%$, so the effect of bardriven injection of intergalactic matter into galactic halos might be present with relative contributions to the average mass of these barred galaxies lower than $20 \%$.
\end{abstract}

Key words. galaxies: kinematics and dynamics - intergalactic medium - galaxies: evolution - galaxies: halos - galaxies: statistics

\section{Introduction}

Intergalactic matter (IGM) is still a field of astrophysics with many open questions and few answers. There are either theoretical arguments or interpretations of observations that suggest the existence of an IGM, although the definitive details of important parameters such as the mean density, whether continuous or in the form of clouds, etc. are not clear yet. Cold dark matter models predict substructure within galactic- and clustermass halos that form in a hierarchical accretion Universe (Klypin et al. 1999; Moore et al. 1999) that could constitute some of the clumpy structures in the IGM. Indeed, stellar and cold gas in galaxies contribute $8\left({ }_{-5}^{+4}\right) \%$ of the total amount of the Big Bang-produced baryonic matter predictions (Bell et al. 2003); this implies a low overall efficiency of galaxy formation, and that the rest of the baryonic material must be some way away from the visible galaxies, possibly in the IGM. Some interstellar gas is ejected into the IGM by tidal interactions between galaxies (Morris \& van den Bergh 1994), SN explosions, etc. Of course, the existence of intracluster gas in rich and irregular clusters, which have diffuse emission and X-ray emission associated with hot gas, is well established. Possibly, some high-velocity clouds (HVCs) observed in the radio are located outside our Galaxy, i.e. belonging to the IGM (Blitz et al. 1999; Braun \& Burton 1999). There is also a large number of lines visible in optical at high redshifts or UV in low redshifts - the Lyman- $\alpha$ forest - which are presumably caused by clouds along the line of sight of QSOs (Rauch 1998).

There is a need for a substantial accretion of low angular momentum material from the IGM into the galaxies (Fraternali et al. 2007). Chemical evolution in the local Galactic disc (the G-dwarf problem, ${ }^{9} \mathrm{Be}$ abundance and others;
Rocha-Pinto \& Maciel 1996; Casuso \& Beckman 1997) needs a continual but episodic infall of metal-poor gas that mixes slowly with the rest of the interstellar medium. López-Corredoira et al. (1999) deduce that the IGM mass must currently represent at least around one half of the total mass of the Local Group, given that the accretion rate, as inferred from chemical evolution, has not decreased significantly during the disc lifetime.

The existence of matter in the IGM should have some effects on the formation and evolution of the galaxies. For instance, it is a possible explanation for the formation of warps in spiral galaxies (López-Corredoira et al. 2002a). It is also, as said, a likely explanation for the chemical evolution of galaxies (Casuso \& Beckman 1997). Here, we study another effect: the accretion of this IGM matter due to dynamical friction. It is clear that the disc mass might be fed by the collisional friction of baryonic matter with disc gas. The enrichment of the halo by means of a gravitational interaction is instead the topic to be treated here. In particular, the component of the galaxy responsible of this gravitational interaction will be the bar.

Bar-halo interactions have been studied for several reasons. For instance, as a mechanism of angular momentum transfer. It is well established that strong bars rotating in dense halos generally slow down as they lose angular momentum to the halo through dynamical friction (Debattista \& Sellwood 1998, 2000; Athanassoula 2003, 2005). This friction can be avoided or found to be anomalously weak in some circumstances and bar slowdown can be delayed for a period in a metastable state (Sellwood \& Debattista 2006), although Sellwood \& Debattista (2006) demonstrate that mild external, or internal, perturbations quickly restore the usual frictional drag; it is therefore unlikely that a strong bar in a galaxy having a dense halo could rotate 
for a long period without friction. A more long-lasting effect to be considered is that the velocity dispersion of the halo particles is high enough to stop the resonances from absorbing considerable amounts of angular momentum (Athanassoula 2003), hence prohibiting bar slowdown. Owing to this angular momentum transfer and self-consistent re-equilibration, strong realistic bars will modify the cusp profile, lowering the central densities within about $30 \%$ of the bar radius in a few bar orbits (Weinberg \& Katz 2007).

In this paper, another effect of the bar will be explored, also using the dynamical friction of this kind of gravitational interaction: the influence of the bar to produce the accretion of IGM matter (initially non-gravitationally linked to the galaxy) into the halo. That is, the interaction with the bar will produce the loss of energy of some particles that cross the galaxy and that will be trapped in it, since they do not have escape velocity, to form part of the galactic halo. This mechanism applies to both baryonic and non-baryonic matter because the friction is purely gravitational. In Sect. 2 this loss of energy and the amount of accreted matter depending on IGM parameters for a Milky-Waylike galaxy is calculated semianalytically. In Sect. 3, it is explained that other factors of the non-conservative potential, for example approaches to individual stars, produce a much lower effect that is totally negligible. In Sect. 4, statistics with observational data are performed in order to see whether barred galaxies have larger masses.

\section{Variations of energy in a barred potential}

We analyse the variations of the energy in particles that follow orbits in certain kinds of non-conservative potentials: those in which the gravitational force varies with time. This happens, for instance, in a barred galaxy, because the rotation of a nonaxisymmetric structure produces variations in the potential due to the bar with respect to an inertial frame. However, the rotating disc has a stationary potential since the axial distribution of mass does not change. If the bar rotates with angular velocity $\omega$, the variation in the energy of a particle along its path (orbit) is:

$$
\begin{aligned}
\Delta E & =\int_{\text {orbit }} \boldsymbol{F}(R, \phi-\omega t, z) \mathrm{d} \boldsymbol{r} \\
& =\int_{\text {orbit }} \boldsymbol{F}(R, \phi-\omega t, z)\left(\mathrm{d} R \boldsymbol{e}_{\boldsymbol{R}}+R \mathrm{~d} \phi \boldsymbol{e}_{\phi}+\mathrm{d} z \boldsymbol{k}\right),
\end{aligned}
$$

in cylindrical coordinates, where $\boldsymbol{F}$ is the gravitational force produced by the bar. If we change to the variable $\phi^{\prime}=\phi-\omega t$ (that is, we adopt the frame that is moving with the bar):

$\Delta E=\int_{\text {orbit }} \boldsymbol{F}\left(R, \phi^{\prime}, z\right)\left(\mathrm{d} R \boldsymbol{e}_{\boldsymbol{R}}+R \mathrm{~d} \phi^{\prime} \boldsymbol{e}_{\phi^{\prime}}+\mathrm{d} z \boldsymbol{k}+R \omega \mathrm{d} t \boldsymbol{e}_{\phi^{\prime}}\right)$.

If the orbits of particles begin at infinite distance and finish at infinite distance:

$\int_{r=\infty, \text { orbit }}^{r=\infty} \boldsymbol{F}\left(\boldsymbol{r}^{\prime}\right) \mathrm{d} \boldsymbol{r}^{\prime}=0$ (because it does not depend on time), (3)

$\Delta E=\omega \int_{r=\infty, \text { orbit }}^{r=\infty} F_{\phi^{\prime}}\left(R, \phi^{\prime}, z\right) R \mathrm{~d} t=\omega \Delta J_{z}$.

The Jacobi integral

$H=E-\omega J_{z}$

is conserved.
Let us consider a monodimensional bar. This is an approximate description of real 3D-bars because the major axis is considerably larger than the other axis - otherwise, they would not be bars - as can be observed, for instance, in the galaxies NGC 1300, NGC 7479, NGC 4123, NGC 1433, NGC 4999, etc. (Sandage \& Bedke 1994) or in our own Galaxy (López-Corredoira et al. 2001, 2007). The mass density distribution will be $\lambda(l)$ as a function of the distance $l$ to its centre. The azimuthal component of the gravitational force produced by this bar of radius $R_{0}$ on a particle of mass $m$ will be:

$F_{\phi}\left(R, \phi^{\prime}, z\right)=-G m \int_{-R_{0}}^{R_{0}} \mathrm{~d} l \frac{\lambda(l) l \sin \phi^{\prime}}{\left(z^{2}+R^{2}+l^{2}-2 R l \cos \phi^{\prime}\right)^{3 / 2}}$.

If we assume $\lambda(l)=$ constant $=\lambda$, the integral can be solved:

$F_{\phi}\left[\boldsymbol{r}^{\prime}\left(R, \phi^{\prime}, z\right)\right]=\frac{-G m \lambda \sin \phi^{\prime}}{z^{2}+R^{2}\left(1-\cos ^{2} \phi^{\prime}\right)}$

$\times\left(r_{+}-\frac{R_{0}^{2}}{r_{+}}+\frac{R_{0} R \cos \phi^{\prime}}{r_{+}}-r_{-}+\frac{R_{0}^{2}}{r_{-}}+\frac{R_{0} R \cos \phi^{\prime}}{r_{-}}\right)$

$r_{+} \equiv\left|\boldsymbol{r}^{\prime}-\boldsymbol{R}_{\mathbf{0}}\right| ; r_{-} \equiv\left|\boldsymbol{r}^{\prime}+\boldsymbol{R}_{\mathbf{0}}\right| ; \boldsymbol{R}_{\mathbf{0}} \equiv R_{0} \boldsymbol{i}$

Therefore, if we want to calculate the gain/loss of energy of the particle, we must just know its orbit $\boldsymbol{r}(t)$ and use expressions (4) and (6) to calculate $\Delta E$.

\subsection{Numerical calculation of orbits}

For our problem, we use semianalytical calculations. The solution of Eqs. (4), (6) is calculated numerically, and the orbit of each particle itself is not solved numerically as an outcome of a numerical simulation in which all particles interact with each other but as the numerical integration of its motion equation. Indeed, we can consider the gravitational potential of the infalling particles as due only to the components of the galaxies and neglect the interaction due to other IGM particles.

Analytically, we cannot calculate the orbit of a particle in a galactic potential; the integrals are too complex to find the solution. But we can calculate $\boldsymbol{r}(t)$ simply by numerical calculation of the differential equation $\ddot{\boldsymbol{r}}=\boldsymbol{a}_{\text {galaxy }}$, the total acceleration produced by the total potential of the galaxy. It is then straightforward to obtain $\boldsymbol{r}^{\prime}(t)$ (the position in the frame that is moving with the bar) from $\boldsymbol{r}(t)$ by using $\phi^{\prime}=\phi-\omega t$. In this case, of course, for the calculation of the orbit, all the potentials are to be taken into account not only the potential of the bar. We can approximate this potential as the sum of several components:

$\boldsymbol{a}_{\text {galaxy }}=\boldsymbol{a}_{\text {halo }}+\boldsymbol{a}_{\mathrm{disc}}+\boldsymbol{a}_{\mathrm{bar}}$.

We give analytical expressions for these acceleration terms as integrals. All these integrals will be performed numerically with the Simpson algorithm, and the solution to the differential equation $\ddot{\boldsymbol{r}}=\boldsymbol{a}_{\text {galaxy }}$ will be carried out with a variable step depending on the acceleration:

$\Delta t=\frac{50 s}{a\left(\mathrm{~m} / \mathrm{s}^{2}\right)+10^{-12}}$.

The Jacobi integral $H$ is conserved for each orbit within variations of $\sim 0.1 \%$. The accuracy in the orbit calculation was also checked by testing that the orbit is approximately the same when decreasing this step $\Delta t$ by a factor of $50\left(\Delta t=\frac{1 \mathrm{~s}}{a\left(\mathrm{~m} / \mathrm{s}^{2}\right)+10^{-12}}\right)$ : the difference in the position of both calculations after a travelled 
path of more than $1000 \mathrm{kpc}$ (once the particle fell from a distance $r_{0}$ towards the centre and, after that, it crosses the shell at $r=r_{0}$; with $r_{0}=400-500 \mathrm{kpc}$ ) is $\sim 0.4 \mathrm{kpc}$ on average. These errors are not important for the present calculations because we do not require too much accuracy in the orbit; it is only used for the path integral of Eq. (4). Moreover, a small random error added to the orbit is equivalent to a small random variation of the initial conditions, which in comparison with the range of impact parameters $(0-200 \mathrm{kpc})$ is negligible.

\subsubsection{Halo potential}

We approximate the halo as spherical, i.e. we neglect the ellipticity:

$\boldsymbol{a}_{\text {halo }}(\boldsymbol{r})=-\frac{\left[4 \pi G \int_{0}^{r} \mathrm{~d} t t^{2} \rho_{\text {halo }}(t)\right] \boldsymbol{r}}{r^{3}}$.

We then use a truncated flat model by Battaglia et al. (2005):

$\rho_{\text {halo }}(r)=\frac{M_{\text {halo }}}{4 \pi} \frac{a^{2}}{r^{2}\left(r^{2}+a^{2}\right)^{3 / 2}}$.

\subsubsection{Disc potential}

For the disc, we use a typical exponential function considering its thickness to be negligible. In order to reduce the calculation to only one integral, which reduces the calculation time very considerably, we express it in elliptic integrals (Binney \& Tremaine 1987, Eq. (2-142b)):

$\boldsymbol{a}_{\mathrm{disc}}[\boldsymbol{r}(R, \phi, z)]=-\frac{\partial \phi_{\mathrm{disc}}}{\partial R} \boldsymbol{e}_{\boldsymbol{R}}-\frac{\partial \phi_{\mathrm{disc}}}{\partial z} \boldsymbol{k}$,

$$
\begin{aligned}
\phi_{\text {disc }} & =-\int_{0}^{\infty} \mathrm{d} T T \int_{0}^{2 \pi} \mathrm{d} \alpha \frac{G \sigma(T)}{\sqrt{z^{2}+R^{2}+T^{2}-2 R T \cos (\phi-\alpha)}} \\
& =\frac{-2 G}{\sqrt{R}} \int_{0}^{\infty} \mathrm{d} T K(k) k \sigma(T) \sqrt{T},
\end{aligned}
$$

where

$k(R, T, z)=\sqrt{\frac{4 R T}{z^{2}+(R+T)^{2}}}$,

and $K(k)$ are the complete elliptic integrals of the first kind,

$K(k)=\int_{0}^{1} \frac{\mathrm{d} t}{\sqrt{\left(1-t^{2}\right)\left(1-k^{2} t^{2}\right)}}$.

The two derivatives of $\phi$ are:

$$
\begin{aligned}
& \frac{\partial \phi_{\text {disc }}}{\partial R}=\frac{G}{R^{3 / 2}} \int_{0}^{\infty} \mathrm{d} T \\
& \times\left[K(k)-\frac{1}{4}\left(\frac{k^{2}}{1-k^{2}}\right)\left(\frac{T}{R}-\frac{R}{T}+\frac{z^{2}}{R T}\right) E(k)\right] k \sigma(T) \sqrt{T}
\end{aligned}
$$

(Binney \& Tremaine 1987, Eq. (2-146)), and

$$
\frac{\partial \phi_{\mathrm{disc}}}{\partial z}=\frac{G z}{2 R^{3 / 2}} \int_{0}^{\infty} \mathrm{d} T \frac{k^{3} E(k)}{1-k^{2}} \frac{\sigma(T)}{\sqrt{T}},
$$

where $E(k)$ are the complete elliptic integrals of the second kind,

$$
E(k)=\int_{0}^{1} \mathrm{~d} t \sqrt{\frac{1-k^{2} t^{2}}{1-t^{2}}} .
$$

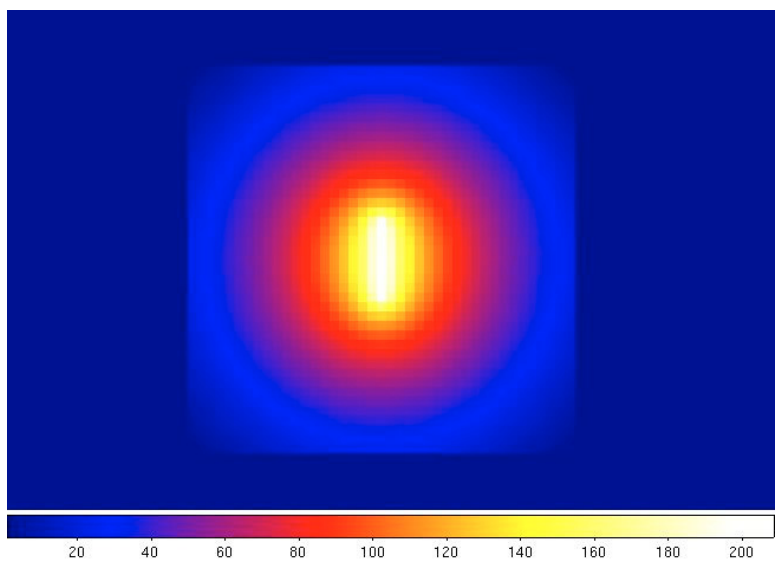

Fig. 1. Modulus of the acceleration in a monodimensional bar potential with the parameters of Sect. 2.1.4. Logarithmic scale. Range of $x$ and $y$ between $-20 \mathrm{kpc}$ and $20 \mathrm{kpc}$.

We assume an exponential disc such that:

$\sigma(R)=\sigma_{0} \exp \left(-\frac{R}{h_{R}}\right)$

\subsubsection{Bar potential}

For the bar, as said, we adopt a monodimensional structure rotating with angular velocity $\omega$ :

$\boldsymbol{a}_{\mathrm{bar}}=-G \int_{-R_{0}}^{R_{0}} \mathrm{~d} l \frac{\lambda(l)[\boldsymbol{r}-l[\cos (\omega t) \boldsymbol{i}+\sin (\omega t) \boldsymbol{j}]]}{\left(z^{2}+R^{2}+l^{2}-2 R l \cos (\phi-\omega t)\right)^{3 / 2}}$.

Other bar potentials could be used (e.g., Athanassoula 1992) but this one is used for its simplicity. The IGM particles will never cross this $1 \mathrm{D}$ structure, because there is zero probability that the path by chance crosses the line of the bar, so this potential will not give problems of divergence. The potential also behaves well at large distance, as shown in Fig. 1, the acceleration falling as $\propto r^{-2}$.

\subsubsection{Parameters for our numerical calculations}

The parameters that we used are those more or less typical for Milky Way-like galaxies.

A total mass for the halo $M_{\text {halo }}=1.2 \times 10^{12} M_{\odot}$, and $a=105 \mathrm{kpc}$ (Battaglia et al. 2005). The mass within a radius $r$ would be $M(r)=M_{\text {halo }} \frac{r}{\sqrt{r^{2}+a^{2}}} \cdot 96.7 \%$ of the halo mass is within $r<400 \mathrm{kpc}$, so it can be approximated for $r>400 \mathrm{kpc}$ as a Keplerian potential of mass equal to $M_{\text {halo }}$ (plus the mass of the disc and the bar).

For the disc, we adopt $\sigma_{0}=1 \mathrm{~kg} / \mathrm{m}^{2}$ (in agreement with the solar neighbourhood measurements by Kuijken \& Gilmore 1989 ) and $h_{R}=3.4 \mathrm{kpc}$ (a typical value in a Milky Way-like galaxy; López-Corredoira et al. 2002b). The corresponding total mass of the disc is $3.5 \times 10^{10} M_{\odot}$.

For the bar, we assume a total mass of $1.7 \times 10^{10} M_{\odot}$ (Sevenster et al. 1999), more or less in agreement with Kuijken \& Dubinski's (1995) self-consistent models of the Galaxy. This gives a constant distribution $\lambda(l)=1.4 \times 10^{20} \mathrm{~kg} / \mathrm{m}$ for a radius $R_{0}=4 \mathrm{kpc}$ (estimated value of the long-bar radius in our Galaxy, López-Corredoira et al. 2001, 2007). This mass of the long bar is indeed not well known (López-Corredoira et al. 2007) and the bar of Sevenster et al. (1999) is 35\% shorter (the axial 
ratio difference is not so important, as we will see below) than López-Corredoira et al. (2007) bar, so we are not setting the parameters of the bar accurately, but as a more or less correct order of magnitude (see further discussion in Sect. 2.6 of what would happen with more or less massive bars).

The angular rotation velocity, $\omega$, is taken as $1.9 \times 10^{-15} \mathrm{~s}^{-1}$ (as measured in the Milky Way; Debattista et al. 2002). Most strong bars in real galaxies appear to rotate rapidly (Aguerri et al. 2003), so it is not expected that this velocity will slow down too much. Although the dynamical halo-bar friction predicts a quick slowdown in this velocity (Debattista \& Sellwood 1998, 2000; Athanassoula 2003, 2005), there might be a metastable state in which the bar-halo also remains a long time without friction (Sellwood \& Debattista 2006), or a state in which the velocity dispersion of the halo particles is high, so as to stop the resonances from absorbing considerable amounts of angular momentum (Athanassoula 2003), thus prohibiting bar slow down. Whatever the explanation, the fact of fast bars in most barred galaxies remains, and we therefore do not consider any friction or change in $\omega$ with time.

With these parameters, the stationary (Lagrangian) points of the Galactic potential are at: L1, L2: $\phi_{L}^{\prime}=0, \pi, z=0$, $R_{L}=5.25 \mathrm{kpc}$; L4, L5: $\phi_{L}^{\prime}=\pi / 2,3 \pi / 2, z=0, R_{L}=4.59 \mathrm{kpc}$ (for more information about the Lagrangian points in a barred potential see Pfenninger 1990 or Athanassoula 1992), whose Jacobi integrals are: $H=-1.41 \times 10^{11} \mathrm{~J}$ and $-1.29 \times 10^{11} \mathrm{~J}$ respectively. The fact that the Lagrangian points L4, L5 are $13 \%$ closer to the centre than the Lagrangian points L1, L2 is expected for average strong bars; the difference is typically $6 \%$ for average bar models according to Athanassoula (1992) but it may be as large as $20 \%$ for the strongest bars. The strength of the bar can be compared with another parameter defined by Athanassoula (1992): half of the quadrupole moment along its major axis

$Q_{m}=\frac{1}{5} M_{\mathrm{bar}} R_{0}^{2}$.

This expression is derived from Eq. (5) of Athanassoula with $a=R_{0}, b=0$ (infinite axial ratio $\left.a / b\right), n=0$ (constant density along the bar). With our parameters we get $Q_{m}=5.4 \times 10^{4}$ (in units $10^{6} M_{\odot} \mathrm{kpc}^{2}$, as in Athanassoula 1992). This is again an indication that the strength of our bar is a typical one within strong bars (weak bars have $Q_{m} \sim 10^{4}$, very strong bars have $Q_{m} \sim 10^{5}$; Athanassoula 1992). It is also remarkable that the infinite axial ratio $\mathrm{a} / \mathrm{b}$ in a monodimensional bar does not significantly alter the effects of a bar with finite but high axial ratios since $Q_{m} \propto\left(a^{2}-b^{2}\right)$ (Athanassoula 1992, Eq. (5)) and $\left(a^{2}-b^{2}\right) \approx a^{2}$ for high $a / b$, either finite or infinite.

\subsubsection{Initial conditions of the IGM particles}

We assume now that our galaxy is moving with respect to the IGM (or the IGM is moving with respect to the galaxy). This hypothesis was already used in López-Corredoira et al. (2002a) as an explanation to produce warps in disc galaxies and now will be explored just to calculate the fraction of the particles that are gravitationally trapped in the galactic potential due to a negative energy variation. Basically, the model consists of a constant density $\left(\rho_{\infty}\right)$ medium which has at infinite distance from the galaxy an average velocity $\boldsymbol{v}_{\infty}$ with respect to the centre of galaxy, and an angle of $\boldsymbol{v}_{\infty}$ with respect to the plane of the galaxy equal to $\theta_{\infty}$. We neglect the effect of the dispersion of velocities with respect to this average velocity.

We randomly distribute the values of $b$ (impact parameter with respect to the centre of the galaxy), $\phi_{b}$ (azimuthal angle in cylindrical coordinates with the vector $\boldsymbol{v}_{\infty}$ as vertical axis) following the conditions for a homogeneous distribution, equivalent to do integrals in the way $\int_{0}^{2 \pi} \mathrm{d} \phi_{b} \int_{0}^{b_{\max }} \mathrm{d} b b$ (..). We set $b_{\max }=200 \mathrm{kpc}$. That is, we neglect the contribution of particles whose impact parameter is larger than $200 \mathrm{kpc}$; this is justified because, as we shall see in following subsections, the particles which lose more energy have lower value of $b$, except in cases with low $v_{\infty}$.

Since an infinite distance cannot be introduced in the computer (integration of the orbit would take an infinite time), we must begin the integration from a finite distance, which we choose to be at $400 \mathrm{kpc}<r_{0}<500 \mathrm{kpc}$. We distribute our particles randomly at a shell with this range of radii. The distribution with different $r_{0}$ is useful to integrate over different positions of the bar when the particle reaches the galactic disc; that is, it is equivalent to doing an integration with different initial bar angles $\omega\left(t_{0}\right)$. The time to cross $100 \mathrm{kpc}$ at around $70 \mathrm{~km} \mathrm{~s}^{-1}$ (the velocity of the particle at radius $r_{0}$ assuming $v_{\infty}=50 \mathrm{~km} \mathrm{~s}^{-1}$ ) is $1.4 \mathrm{Gyr}$, so doing the integration between $400 \mathrm{kpc}<r_{0}<$ $500 \mathrm{kpc}$ is equivalent to doing an integration over a time of $1.4 \mathrm{Gyr}$, equivalent to 14 cycles of the bar (one cycle/100 Myr); moreover, there is an added dispersion in the position of the bar when the particle reaches the centre of the galaxy because the different values of $\phi_{b}$ and $b$ get different infall times to reach the centre, so in practice we are integrating over a range larger than 1.4 Gyr. This ensures that all angles of the bar are covered more or less uniformly (departures from uniformity are negligible and less important than Poissonian or other fluctuations). In this way, we are doing a Monte Carlo simulation over the variables $b, \phi_{b}$ and $r_{0}$.

At the distance of 400-500 kpc the non-monopolar terms of the gravitational potential are negligible and almost the entire mass of the galaxy is within this radius, so the potential can be approximated as one produced by a point-like source in the centre of the galaxy with its total mass. Nonetheless, this potential is not totally negligible at these distances, so we must correct the initial conditions with respect to the conditions at infinite distance:

$\boldsymbol{r}_{\mathbf{0}}=-\sqrt{r_{0}-r_{\mathrm{imp}}^{2}} \boldsymbol{e}_{v_{\infty}}+r_{\mathrm{imp}} \boldsymbol{e}_{b_{\infty}}$

where $\boldsymbol{e}_{v_{\infty}}$ is the unit vector parallel to the initial velocity at infinity, and $\boldsymbol{e}_{b_{\infty}}$ is the vector perpendicular to $\boldsymbol{e}_{v_{\infty}}$ in the plane perpendicular to the one defined by $\boldsymbol{r}_{\mathbf{0}}$ and $\boldsymbol{v}_{\infty}$, i.e. the direction of the impact parameter, pointing towards an increase in $b$.

$\boldsymbol{v}_{\mathbf{0}}=v_{0} \cos \left(\alpha-\alpha_{0}\right) \boldsymbol{e}_{v_{\infty}}-v_{0} \sin \left(\alpha-\alpha_{0}\right) \boldsymbol{e}_{b_{\infty}}$.

The parameters $v_{0}, r_{\text {imp }}$ and $\alpha, \alpha_{0}$ are derived from the analytical expressions of a hyperbolic orbit (see López-Corredoira et al. 2002a; Sect. 3.3) and taking into account that the angular momentum is constant along the orbit:

$v_{0}=\sqrt{\frac{2 G M\left(r_{0}\right)}{r_{0}}+v_{\infty}^{2},}$
$r_{\text {imp }}=r_{0}\left|\sin \left[\beta-\cos ^{-1}(-1 / \epsilon)\right]\right|$,

$\beta=\cos ^{-1}\left[\frac{\left(\frac{\epsilon}{A r_{0}}-1\right)}{\epsilon}\right]$,

$A=\frac{v_{\infty}^{2}}{G M\left(r_{0}\right)} \frac{\epsilon}{\epsilon^{2}-1}$, 
$\epsilon=\sqrt{1+\left(\frac{b v_{\infty}^{2}}{G M\left(r_{0}\right)}\right)^{2}}$

$\alpha_{0}=\frac{\pi}{2}+\cos ^{-1}\left(\frac{r_{\text {imp }}}{r_{0}}\right)$ (defined to be between $\pi / 2$ and $\left.\pi\right)$,

$\alpha=\pi-\sin ^{-1}\left(\frac{b v_{\infty}}{r_{0} v_{0}}\right) \quad($ defined to be between $\pi / 2$ and $\pi)$.

The results of the orbit with these analytical expressions were compared with the results of numerical calculations, and they agree. By applying these formulae, we do not need to do numerical calculations beyond 400-500 kpc.

\subsection{Histogram of energy variations}

The only free parameters for the calculation of the energy variation of each particle once the galactic parameters are established are $\rho_{\infty}, v_{\infty}$ and $\theta_{\infty}$. We could also consider $M_{\text {halo }}$ as a third free parameter instead of a fixed value of $M_{\text {halo }}=1.2 \times 10^{12} M_{\odot}$, since the total mass of the halo might be variable with the time, precisely because of the accreting mechanism proposed in this paper: the loss of energy of some particles which are trapped when this loss of energy is higher than

$E_{\infty}=\frac{1}{2} m v_{\infty}^{2}$.

As an example, let us set $v_{\infty}=50 \mathrm{~km} \mathrm{~s}^{-1}$ (a typical value for the peculiar motion of a galaxy) and $\theta_{\infty}=45^{\circ}$, with $M_{\text {halo }}=$ $1.2 \times 10^{12} M_{\odot}$. The results for 10000 particles are in Figs. 2-4, which show that the loss of energy is high enough to be trapped only for $b<100 \mathrm{kpc}$ or $-2.2 \times 10^{11}<H<1.2 \times 10^{11} \mathrm{~J}$ (for comparison, the Jacobi integrals of the Lagrangian points are $H=-1.41 \times 10^{11} \mathrm{~J}$ and $\left.-1.29 \times 10^{11} \mathrm{~J}\right)$, and only 426 of the 10000 particles lose an energy higher than $E_{\infty}$. Equations (4) and (6) were applied to calculated $\Delta E$ for each particle once it moves away from the galaxy. There are, as observed in Fig. 2, practically the same number of particles which gain $E \mathrm{~d} E$ as those which lose $E \mathrm{~d} E$.

Let $R_{200}$ be the ratio of particles within $b<200 \mathrm{kpc}$ which are trapped by the halo. According to the above, this is $R_{200}=$ $\frac{426}{10000}=0.0426 \pm 0.0021$. Hence, the accretion ratio will be:

$$
\begin{aligned}
\frac{\mathrm{d} M_{\text {halo }}}{\mathrm{d} t} & =\rho_{\infty} v_{\infty} R_{200} \int_{0}^{2 \pi} \mathrm{d} \phi_{b} \int_{0}^{200 \mathrm{kpc}} \mathrm{d} b b \\
& =1.9 \times 10^{24} \rho_{\infty}\left(\mathrm{kg} / \mathrm{m}^{3}\right) v_{\infty}\left(\mathrm{km} \mathrm{s}^{-1}\right) R_{200} M_{\odot} / \mathrm{yr} \\
& =4.0( \pm 0.2) \times 10^{24} \rho_{\infty}\left(\mathrm{kg} / \mathrm{m}^{3}\right) M_{\odot} / \mathrm{yr} .
\end{aligned}
$$

\subsubsection{Dependence on the initial infall angle}

The dependence on the angle $\theta_{\infty}$ is illustrated in Fig. 5. The $R_{200}$ ratios are: $0.0441( \pm 0.0021)$ for $\theta_{\infty}=5^{\circ}, 0.0436( \pm 0.0021)$ for $\theta_{\infty}=25^{\circ}, 0.0536( \pm 0.0023)$ for $\theta_{\infty}=65^{\circ}$, and $0.1563( \pm 0.0040)$ for $\theta_{\infty}=85^{\circ} \cdot R_{200}$ does not change appreciably except for $\theta_{\infty}=$ $85^{\circ}$, which is $3-4$ times higher. The polar accretion seems to favour the mechanism. The dependence is roughly described by a law:

$$
\begin{gathered}
R_{200}\left(\theta_{\infty} ; v_{\infty}=50 \mathrm{~km} \mathrm{~s}^{-1}, M_{\text {halo }}=1.2 \times 10^{12} M_{\odot}\right) \\
\approx 0.043+0.20 \exp \left[-0.12\left[90-\theta_{\infty}(\mathrm{deg})\right]\right] .
\end{gathered}
$$

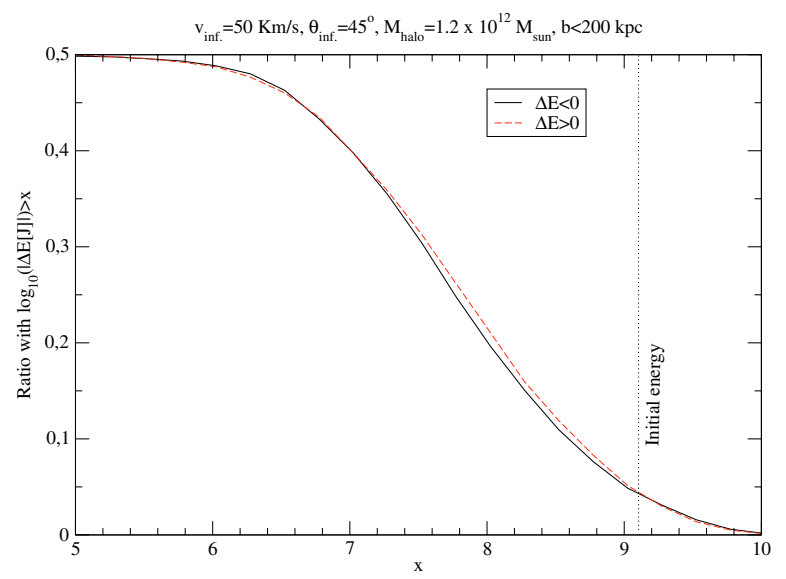

Fig. 2. Histogram of distribution of energy gain/loss per unit mass $(\mathrm{kg})$.

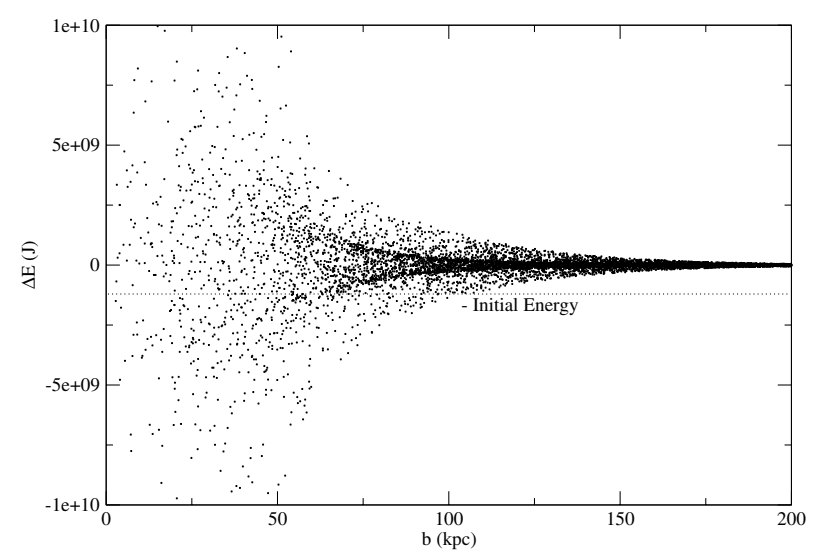

Fig. 3. Energy gain/loss in the simulation of particles with different impact parameter $(b)$.



Fig. 4. Energy gain/loss in the simulation of particles as a function of its Jacobi integral. For comparison, the Jacobi integrals for the Lagrangian points are $H=-1.41 \times 10^{11} \mathrm{~J}$ and $-1.29 \times 10^{10} \mathrm{~J}$.

Since we are interested in calculating the order of magnitude, we can disregard this dependence and leave for our remaining calculations a value of $\theta_{\infty}=45^{\circ}$.

\subsubsection{Dependence on the initial velocity}

However, the dependence on initial velocity is high. The histograms in Fig. 6 show it. The ratios $R_{200}$ are (note that $E_{\infty}$ varies with $\left.v_{\infty}\right)$ : $>0.4966$ for $v_{\infty}=10 \mathrm{~km} \mathrm{~s}^{-1}, 0.2511( \pm 0.0050)$ 


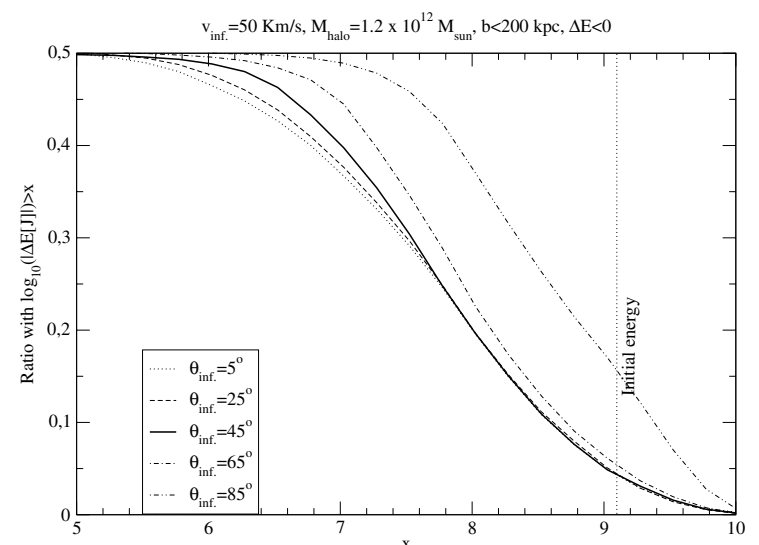

Fig. 5. Histogram of distribution of energy loss as a function of the initial infall angle.



Fig. 6. Histogram of distribution of energy loss as a function of the initial velocity.

for $v_{\infty}=30 \mathrm{~km} \mathrm{~s}^{-1}, 0.0118( \pm 0.0011)$ for $v_{\infty}=70 \mathrm{~km} \mathrm{~s}^{-1}$, $0.0035( \pm 0.0006)$ for $v_{\infty}=90 \mathrm{~km} \mathrm{~s}^{-1}$, and $0.0012( \pm 0.0003)$ for $v_{\infty}=110 \mathrm{~km} \mathrm{~s}^{-1}$. The ratio of higher energy loss is maximum for lower initial velocities, both in terms of absolute energy and relative energy with respect to $E_{\infty}$. The explanation for this is multiple. First, for higher velocities the particles go very fast when they reach the bar, and since $\Delta E$ in Eq. (4) is proportional to the time the particle spends near the bar, the loss of energy is lower. Second, the capacity to attract towards the centre particles with high $b$ is larger for lower values of the initial velocity. Moreover, $E_{\infty}$ is higher for higher initial velocities, so the rate of energy loss is lower too. For the cases $v_{\infty} \leq 30 \mathrm{~km} \mathrm{~s}^{-1}$, it is observed that some particles with $b>200 \mathrm{kpc}$ also lose $\Delta E<-E_{\infty}$; therefore, the trapped mass is even higher than the ratio given in Eq. (32). The dependence is roughly described by the law:

$$
\begin{aligned}
& R_{200}\left(v_{\infty}>\approx 30 \mathrm{~km} \mathrm{~s}^{-1} ; \theta_{\infty}=45^{\circ}, M_{\text {halo }}=1.2 \times 10^{12} M_{\odot}\right) \\
& \approx 0.82 \exp \left[-0.06 v_{\infty}\left(\mathrm{km} \mathrm{s}^{-1}\right)\right] .
\end{aligned}
$$

\subsubsection{Dependence on the halo mass}

The effect of the variation of halo mass is similar to the effect of the variation in the initial velocity because the amount of mass is proportional to the acceleration, and consequently is related to the velocity of the particle. Figure 7 illustrates this. A maximum accretion is obtained for the largest mass. Lower

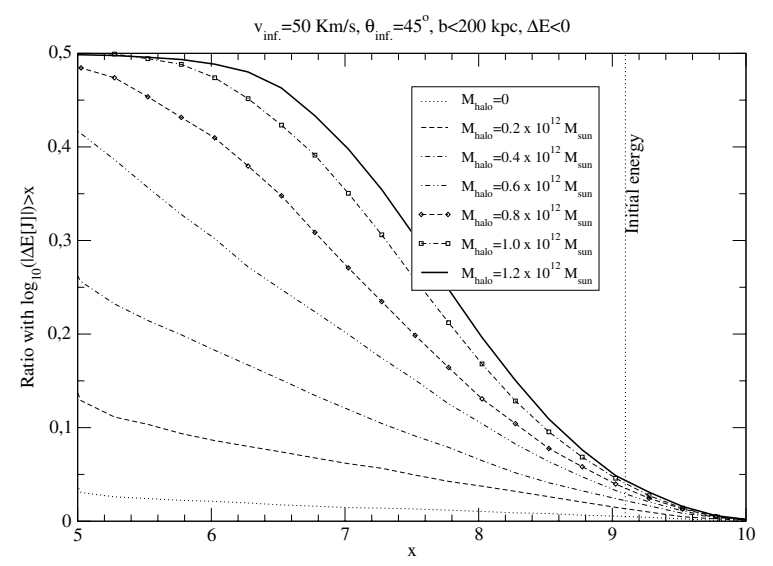

Fig. 7. Histogram of distribution of energy loss as a function of halo mass.

masses have lower capacities to trap particles with high $b$. The values of $R_{200}$ obtained are: $0.0049( \pm 0.0007)$ for $M_{\text {halo }}=0$, $0.0092( \pm 0.0010)$ for $M_{\text {halo }}=0.2 \times 10^{12} M_{\odot}, 0.0220( \pm 0.0015)$ for $M_{\text {halo }}=0.4 \times 10^{12} M_{\odot}, 0.0299( \pm 0.0017)$ for $M_{\text {halo }}=$ $0.6 \times 10^{12} M_{\odot}, 0.0362( \pm 0.0019)$ for $M_{\text {halo }}=0.8 \times 10^{12} M_{\odot}$, and $0.0396( \pm 0.0020)$ for $M_{\text {halo }}=1.0 \times 10^{12} M_{\odot}$. It is clear that it is proportional to the halo mass, roughly according to:

$$
\begin{array}{r}
R_{200}\left(M_{\text {halo }} ; \theta_{\infty}=45^{\circ}, v_{\infty}=50 \mathrm{~km} \mathrm{~s}^{-1}\right) \\
\approx 0.0062+0.034 M_{\text {halo }}\left(10^{12} M_{\odot}\right) .
\end{array}
$$

\subsection{Other ways of accretion and the escape of halo particles}

The gain or loss of mass in the halo due to other mechanisms will not be considered in this paper, but only the mechanism related to the dynamical friction with the bar. There are other mechanisms of accretion, such as friction with the disc of particles or clouds that cross it. There are also ways for the halo to lose mass, such as galaxy stripping in interactions with other galaxies or the IGM, or the escape of particles when they reach a velocity larger than escape velocity due to multiple interactions. We neglect these other mechanisms because we want to consider only the effect of the bar in increasing the halo mass independently of other effects. The reality is more complex, of course, but here we just consider a toy model for rough estimates of the net effect of an increase in the accretion of mass in barred galaxies with respect to non-barred galaxies.

The non-conservative potential of the bar could also produce an increment of the energy of the halo particles and these could reach the escape velocity producing some kind of "evaporation" of the halo. The mechanism is the same as the one explained in previous subsections but the initial conditions will be different from those explained in Sect. 2.1.5. Indeed, the fact that we get some net accretion of some IGM particles arises because some of them go through the centre of the galaxy, but the virialized halo has totally different initial conditions.

Assuming that the halo particles have an initial velocity corresponding to a circular velocity plus isotropic dispersion velocities with a component in the radial direction (given the isotropy, the dispersion in other directions will be the same; the isotropic representation, while more or less correct in the inner halo, is not correct in the outer halo (Dehnen \& McLaughlin 2005), but we consider it here as a rough approximation)

$\sigma_{r}(r) \propto\left[\rho(r) r^{\alpha}\right]^{1 / 3}$ 
$\alpha=35 / 18$ (Dehnen \& McLaughlin 2005), in agreement with simulations and analytical solutions of self-gravitating dynamical equilibriums; with the halo density of Eq. (11), and $\sigma_{r}(r=$ $a)=v_{\mathrm{c}}(a) / \sqrt{3}\left(\right.$ Dehnen $\&$ McLaughlin 2005), where $v_{\mathrm{c}}(r)$ is the circular velocity, we can perform again the numerical calculation of the orbits and the increment of energy according to Eqs. (4) and (6). The result with 10000 halo particles whose initial conditions are distributed randomly according to the probability densities of positions and velocities including the dispersions, during 14 Gyr orbit calculation, gives the result that none of these particles gets an increase in energy $\Delta E>\frac{G M\left(r_{0}\right)}{2 r_{0}}$, where $r_{0}$ is the initial distance from the centre of the galaxy. This means that the increase in the halo evaporation due to the bar-halo interaction is negligible: $\frac{\Delta M}{M}<\sim 10^{-4}$ during the whole life of the galaxy.

\subsection{Evolution of the halo mass}

Let us suppose that the dependence on the initial angle, initial velocity and the halo mass are separable. This is not strictly correct, but may be appropriate as a rough estimation. In this case, a generalization of the expression (32) will be:

$$
\begin{aligned}
& \frac{\mathrm{d} M_{\text {halo }}}{\mathrm{d} t}=2.1 \times 10^{23} \rho_{\infty}\left(\mathrm{kg} / \mathrm{m}^{3}\right) v_{\infty}\left(\mathrm{km} \mathrm{s}^{-1}\right) \\
& \times \exp \left[-0.06 v_{\infty}\left(\mathrm{km} \mathrm{s}^{-1}\right)\right]\left[1+4.7 \exp \left[-0.12\left[90-\theta_{\infty}(\mathrm{deg})\right]\right]\right] \\
& \times\left[1+5.5 \times 10^{-12} M_{\text {halo }}\left(M_{\odot}\right)\right] M_{\odot} / \mathrm{yr} .
\end{aligned}
$$

Here we are assuming that the disc and the bar do not change with time. The solution of this differential equation will be:

$M_{\text {halo }}(t)=-1.8 \times 10^{11} M_{\odot}+\left[1.8 \times 10^{11} M_{\odot}+M_{\text {halo }}(t=0)\right] \times \mathrm{e}^{E},(38)$

$E=1.2 \times 10^{12} \rho_{\infty}\left(\mathrm{kg} / \mathrm{m}^{3}\right) v_{\infty}\left(\mathrm{km} \mathrm{s}^{-1}\right) \times \exp \left[-0.06 v_{\infty}\left(\mathrm{km} \mathrm{s}^{-1}\right)\right]$

$\times\left[1+5.5 \exp \left[-0.12\left[90-\theta_{\infty}(\mathrm{deg})\right]\right]\right] \times t(\mathrm{yr})$,

and the total accretion in the lifetime of the Galaxy (we take $t=1.4 \times 10^{10} \mathrm{yr}$ ) due to this mechanism is

$\Delta M_{\text {halo }}=\left[1.8 \times 10^{11} M_{\odot}+M_{\text {halo }}(t=0)\right] \times\left[\mathrm{e}^{E}-1\right]$,

$E=1.7 \times 10^{22} \rho_{\infty}\left(\mathrm{kg} / \mathrm{m}^{3}\right) v_{\infty}\left(\mathrm{km} \mathrm{s}^{-1}\right) \times \exp \left[-0.06 v_{\infty}\left(\mathrm{km} \mathrm{s}^{-1}\right)\right]$

$\times\left[1+5.5 \exp \left[-0.12\left[90-\theta_{\infty}(\mathrm{deg})\right]\right]\right]$.

The necessary conditions for a fraction $F$ of the halo mass to be accreted by this mechanism $\left(M_{\text {halo }}(t=0)=(1-F) M_{\text {halo }}(t)\right.$, $\Delta M=F M_{\text {halo }}(t)$; assuming there are no other mechanisms of accretion) are:

$F=\left[1+\frac{1.8 \times 10^{11} M_{\odot}}{M_{\text {halo }}(t)}\right] \times\left(1+\frac{1}{\mathrm{e}^{E}-1}\right)^{-1}$.

Particularly, for $M_{\text {halo }}(t)=1.2 \times 10^{12} M_{\odot}, \theta_{\infty}=45^{\circ}$ and $v_{\infty}=$ $50 \mathrm{~km} \mathrm{~s}^{-1}$,

$F=1.15\left(1+\frac{1}{\exp \left[4.3 \times 10^{22} \rho_{\infty}\left(\mathrm{kg} / \mathrm{m}^{3}\right)\right]-1}\right)^{-1}$.

This expression has sense only for $\rho_{\infty}\left(\mathrm{kg} / \mathrm{m}^{3}\right) \leq 4.7 \times$ $10^{-23} \mathrm{~kg} / \mathrm{m}^{3}(F \leq 1)$, for higher densities the mass of the halo should be larger. In Fig. 8, the values of $F$ for different densities are plotted. Densities of the IGM around $10^{-24} \mathrm{~kg} / \mathrm{m}^{3}$ (including baryonic and non-baryonic matter) are able to produce a significant amount of accretion by means of this mechanism.

In order to have at least $10 \%$ of the total halo matter accreted by this mechanism $(F=0.1)$ it is necessary that (we keep $\theta_{\infty}=$ $\left.45^{\circ}, M_{\text {halo }}(t)=1.2 \times 10^{12} M_{\odot}\right)$

$F>0.1 \Leftrightarrow v_{\infty}\left(\mathrm{km} \mathrm{s}^{-1}\right) \mathrm{e}^{-0.06 v_{\infty}\left(\mathrm{km} \mathrm{s}^{-1}\right)} \rho_{\infty}\left(\mathrm{kg} / \mathrm{m}^{3}\right)>5.3 \times 10^{-24}(42)$

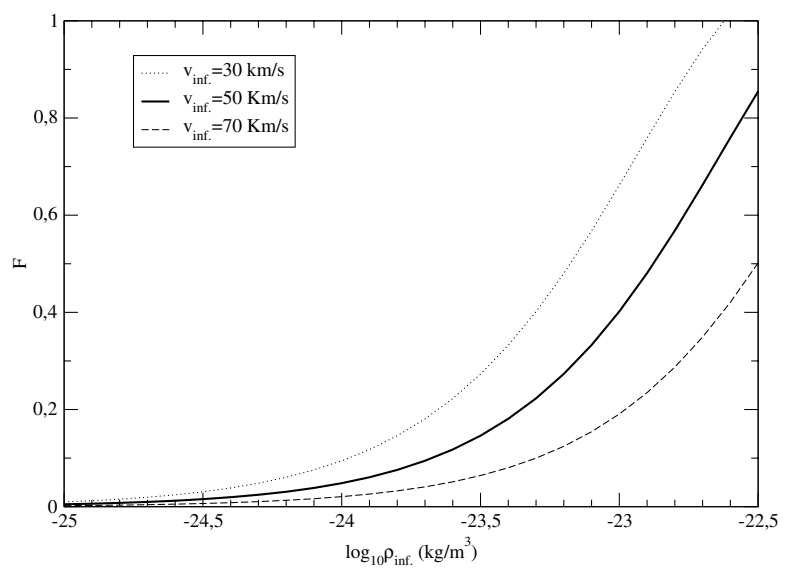

Fig. 8. Fraction of halo mass accreted by means of the mechanism proposed in this paper.

\subsection{Considerations about the range of possible values of the parameters}

\subsubsection{Density of the IGM}

The expression (42) for $F>0.1$ indicates that for $\theta_{\infty}=45^{\circ}$, $M_{\text {halo }}(t)=1.2 \times 10^{12} M_{\odot}, v_{\infty}=50 \mathrm{~km} \mathrm{~s}^{-1}$ we need a density of $2 \times 10^{-24} \mathrm{~kg} / \mathrm{m}^{3}=3 \times 10^{13} M_{\odot} / \mathrm{Mpc}^{3}=10^{2} h_{0}^{-2} \rho_{\text {critical }}$. This is a high density and may be reached by the intracluster gas in some groups or clusters of galaxies, especially in the richest ones, which can have a density much higher than this (including dark matter). The typical IGM densities in clusters are between $10^{-25}$ and $10^{-23} \mathrm{~kg} / \mathrm{m}^{3}$ (Roussel et al. 2000). However, the relative velocities in such environments are also higher, thus decreasing the desired effect (see the next subsection). For cases such as the Local Group this density is too high. The average IGM density of the Local Group is $<\sim 10^{-25} \mathrm{~kg} / \mathrm{m}^{3}$ (assuming a total IGM mass of $<\sim 2 \times 10^{12} M_{\odot}$ (López-Corredoira et al. 1999) in a volume of $\sim 1 \mathrm{Mpc}^{3}$ ).

\subsubsection{Velocity of the IGM}

The product $v_{\infty} \mathrm{e}^{-0.06 v_{\infty}}$ is maximum for $v_{\infty}=17 \mathrm{~km} \mathrm{~s}^{-1}$, at which a density $9 \times 10^{-25} \mathrm{~kg} / \mathrm{m}^{3}$ produces according to Eq. (42) a $F=0.1$. The density might be even lower because, as discussed in Sect. 2.2.2, the accretion of particles with an impact parameter higher than $200 \mathrm{kpc}$ will be considerable (and we are considering in our calculation only particles within $b<200 \mathrm{kpc}$ ). Lower densities are more in the range of possible values of intracluster density in small groups. However, the velocity of $10-20 \mathrm{~km} \mathrm{~s}^{-1}$ is somewhat low. For comparison, the velocity of the centre of the Galaxy with respect to the barycentre of the Local Group is $81 \mathrm{~km} \mathrm{~s}^{-1}$ (Braun \& Burton 1999; Eq. (3)). Maybe in the first stages of the halo formation, when the galactic masses were much lower than $M_{\text {halo }}(t)=1.2 \times 10^{12} M_{\odot}$, the velocities of the galaxies with respect to the average IGM were also lower, since the gravitational potential responsible for such dynamics produced lower accelerations. A more developed study is necessary to clarify the question.

In any case, statistically, it is possible to find a significant fraction of galaxies with low velocities with respect to the average IGM. The present mechanism would apply to these few galaxies. 


\subsubsection{Mass of the halo}

Up to now, we have considered the standard model of galaxies with massive dark matter halos. However, some authors have questioned the very existence of such halos since its evidence is weak on galactic scales (Battaner \& Florido 2000; Sellwood \& Kosowsky 2001; Evans 2001; etc.) and the rotation curves or satellite motions can be explained by alternative hypotheses such as magnetic fields (Battaner \& Florido 2000) or MOND (Sanders $\&$ McGaugh 2002). If we consider a $M_{\text {halo }}(t)=10^{11} M_{\odot}$, necessary to explain the rotation curve of the Galaxy up to $15 \mathrm{kpc}$ (Honma \& Sofue 1996) together with a disc + bar mass of $6 \times 10^{10} M_{\odot}$, we would find from Eq. (40) that (we keep $\theta_{\infty}=$ $\left.45^{\circ}\right)$

$F>0.1 \Leftrightarrow v_{\infty}\left(\mathrm{km} \mathrm{s}^{-1}\right) \mathrm{e}^{-0.1 v_{\infty}\left(\mathrm{km} \mathrm{s}^{-1}\right)} \rho_{\infty}\left(\mathrm{kg} / \mathrm{m}^{3}\right)>8.5 \times 10^{-25}$,

in which the necessary density is 6 times lower than in expression (42).

If the halo mass were still lower than $\sim 10^{11} M_{\odot}$, we could reach the conclusion that most of the halo mass was accreted by this mechanism ( $F$ close to unity). Here, however, we have not included any alternative effects such as magnetic fields or MOND, which would be necessary to make compatible the rotation curves with this low value of the halo.

If the mass of the halo were much higher (Conroy et al. 2005, give typical masses for the halo of the galaxies of $\sim 5 \times 10^{12} M_{\odot}$ ), the relative fraction $F$ would be around $15 \%$ lower than in expression (42), although, of course, the amount of accreted matter is nearly proportional to the halo mass, so in absolute terms the number of accreted solar masses would be higher.

\subsection{Strength of the bar}

In the present paper, we have fixed the parameters of the bar as given in Sect. 2.1.4 which are typical for a normal strong bar: $Q_{m}=5.4 \times 10^{4}$ (in units $10^{6} M_{\odot} \mathrm{kpc}^{2}$, as in Athanassoula 1992). A weak bar would have $Q_{m} \sim 10^{4}$ (Athanassoula 1992). Since the energy loss is proportional to $\lambda$ and, $\lambda$ is proportional to the mass of the bar (Eqs. (4), (6)), it is straightforward to deduce that within the linear regime of accretion $(F \ll 1) F$ will be proportional to mass of the bar. Hence, a weak bar, typically with a mass five times lower than that used here, would reduce $F$ by a factor of five.

\section{Other kinds of dynamical friction}

\subsection{Spiral arms and warps}

As said in Sect. 2, when the gravitational force varies with time, we get a non-conservative potential; that is, a potential in which the energy of the particle in this potential is not constant. Apart from the bar/triaxial bulge, there are other non-axisymmetric structures in the galaxy, which, due to galactic rotation, produce this variation in the potential; for instance, the spiral arms or the warps in the disc. However, these structures normally have a mass much lower than that of the bar (the spiral arm of the Milky Way would have a $\sim 10 \%$ of the disc mass according to the model of Bissantz \& Gerhard 2002; and the mass of the external disc, where the warp is present (López-Corredoira et al. 2002b) is also lower than $10 \%$ of the disc mass); therefore, their effect should be considerably lower, except perhaps in some cases with prominent spiral arms. These cases will not be explored in the present paper.

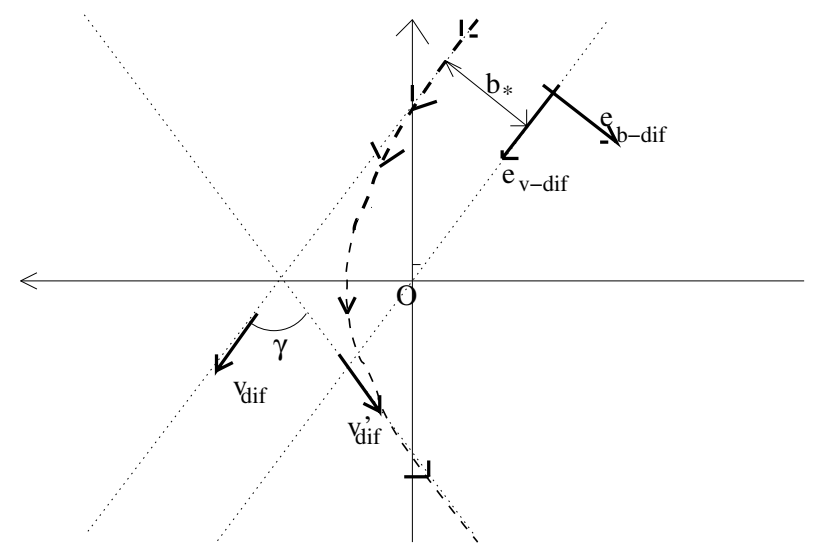

Fig. 9. Graphical representation of the hyperbolic trajectory of a particles which a approach a star (at the centre $\mathrm{O}$ ).

\subsection{Interaction with individual stars}

A way to produce changes in energy is in the interaction of the accreted particles with individual stars. Since individual stars move, the potential of the interaction is not steady, although it is only predominant with respect to the global potential of the whole galaxy when the particle approaches too close to the star. The effect is similar to the exchange of energy of a comet/spacecraft in an approach to a planet the Solar System.

The velocity of a particle when it approaches a star of mass $M_{*}$ with respect to this star is

$\boldsymbol{v}_{\mathrm{dif}}=\boldsymbol{v}-\boldsymbol{v}_{*}$,

where $v$ is the velocity of the particle with respect to the centre of the galaxy, and $\boldsymbol{v}_{*}$ is the rotation(+peculiar) velocity of the star around the centre of the galaxy. The final velocity of the particle when it recedes from the star after the interaction is

$\boldsymbol{v}_{\mathrm{dif}}^{\prime}=\boldsymbol{v}^{\prime}-\boldsymbol{v}_{*}$,

where $\boldsymbol{v}^{\prime}$ is the final velocity with respect to the centre of the galaxy. The star may be considered point-like and the path of the particle in the interaction with the star as a hyperbola with impact parameter $b_{*}$, which follows (see Sect. 3.3 of López-Corredoira et al. 2002a)

$\tan \frac{\gamma}{2}=\frac{G M_{*}}{b_{*} v_{\mathrm{dif}}^{2}}$,

where $\gamma$ is the angle of deviation of the trajectory due to the interaction as represented in Fig. 9.

The final velocity with respect to the star will be:

$\left|\boldsymbol{v}_{\text {dif }}^{\prime}\right|=\left|\boldsymbol{v}_{\text {dif }}\right|$

$\boldsymbol{v}_{\mathrm{dif}}^{\prime}=v_{\mathrm{dif}} \cos (\gamma) \boldsymbol{e}_{v-\mathrm{dif}}+v_{\mathrm{dif}} \sin (\gamma) \boldsymbol{e}_{b-\mathrm{dif}}$,

with the unit vector as specified in Fig. 9, and with respect to the centre of the galaxy:

$$
\begin{aligned}
\boldsymbol{v}^{\prime} & =\boldsymbol{v}_{\mathrm{dif}}^{\prime}+\boldsymbol{v}_{*} \\
& =\left(v_{\mathrm{dif}} \cos \gamma+v_{*} \cos \alpha\right) \boldsymbol{e}_{v-\mathrm{dif}}+\left(v_{\mathrm{dif}} \sin \gamma+v_{*} \sin \alpha\right) \boldsymbol{e}_{b-\mathrm{dif}},
\end{aligned}
$$

$\cos \alpha \equiv \boldsymbol{v}_{*} \boldsymbol{v}_{\mathrm{dif}}$.

Hence,

$v^{\prime}=\sqrt{v_{\mathrm{dif}}^{2}+v_{*}^{2}+2 v_{\mathrm{dif}} v_{*} \cos (\alpha-\gamma)}$, 
Table 1. Comparison of $v_{\max }\left[\mathrm{km} \mathrm{s}^{-1}\right]$ (maximum velocity of rotation curves) and $H W 20 M_{\mathrm{HI}}\left[\mathrm{km} \mathrm{s}^{-1}\right]$ (Half of the HI profile between points where the intensity falls to $20 \%$ of the highest channels in each half of the profile and corrected for relativistic effects; Note: average only with the galaxies which have this datum available) for spiral galaxies in Mathewson et al. (1992) catalogue in barred and non-barred cases as a function of the galaxy type.

\begin{tabular}{lll}
\hline \hline Galaxy type & Non-barred: $(\mathrm{N}),\left\langle v_{\max }\right\rangle,\left\langle H W 20 M_{\mathrm{HI}}\right\rangle$ & Barred: (N), $\left\langle v_{\max }\right\rangle,\left\langle H W 20 M_{\mathrm{HI}}\right\rangle$ \\
\hline+3 & $(339), 174 \pm 3,164 \pm 5$ & $(23), 182 \pm 15,180 \pm 17$ \\
+4 & $(114), 155 \pm 5,156 \pm 7$ & $(10), 205 \pm 17,158 \pm 20$ \\
+5 & $(96), 127 \pm 6,127 \pm 9$ & $(18), 132 \pm 13,125 \pm 13$ \\
+6 & $(526), 150 \pm 2,150 \pm 3$ & $(31), 127 \pm 8,116 \pm 6$ \\
+7 & $(26), 112 \pm 9,117 \pm 13$ & $(8), 77 \pm 7,81 \pm 7$ \\
+8 & $(55), 91 \pm 4,96 \pm 4$ & $(11), 78 \pm 5,85 \pm 5$ \\
\hline
\end{tabular}

while the initial velocity with respect to the centre of the galaxy is

$v=\sqrt{v_{\mathrm{dif}}^{2}+v_{*}^{2}+2 v_{\mathrm{dif}} v_{*} \cos (\alpha)}$.

This implies an increment of the energy of the particle

$\Delta E=\frac{1}{2} m\left(v^{\prime 2}-v^{2}\right)=m v_{\mathrm{dif}} v_{*}[\cos (\alpha-\gamma)-\cos (\alpha)]$.

An approximation for small interactions ( $\gamma \ll 1)$, using (46),

$\Delta E=\frac{1}{2} m\left(v^{\prime 2}-v^{2}\right)=2 m v_{*} \sin \alpha \frac{G M_{*}}{b_{*} v_{\mathrm{dif}}}$.

The particles trapped by the galaxy due to this interaction will be those with $\Delta E<-\frac{1}{2} m v_{\infty}^{2}\left(v_{\infty}\right.$ as the velocity of the particle at infinite distance always is); that is, those particles with impact parameter

$b_{*}<\frac{4 G M_{*} \sin |\alpha| v_{*}}{v_{\mathrm{dif}} v_{\infty}}$,

$\alpha<0$.

For $M_{*}=1 M_{\odot}, v_{*}=200 \mathrm{~km} \mathrm{~s}^{-1}$ (the rotation as the main component), $v_{\infty}=50 \mathrm{~km} \mathrm{~s}^{-1}$, for an average case of $\alpha=-45^{\circ}$ and $v_{\text {dif }} \sim 100 \mathrm{~km} \mathrm{~s}^{-1}$, the constraint is $b_{*}<\sim 2$ AU. With the typical surface density in the galactic disc of $10-100 \mathrm{star} / \mathrm{pc}^{2}$ (López-Corredoira et al. 2002b), the probability of a particle having an impact parameter less than $2 \mathrm{AU}$ is $\sim 3-30 \times 10^{-9}$. This is just a rough estimate but the order of magnitude should be more or less correct. Therefore, the accretion ratio is much lower than the values of $R_{200}$ obtained in Sect. 2.2, and a much lower accretion (totally negligible) is expected to be produced in terms of the star/particle interaction.

\section{Comparison with observations: the effect of the bar on the halo mass}

It is not straightforward to check this effect on real galaxies. We would need to know the complete history of each galaxy, the epoch of formation of its bar and other characteristics which are not directly available. Nonetheless, we can analyse whether spiral galaxies with a prominent bar have different rotation-curve amplitudes from the same type of galaxies without bar. Indeed, this analysis cannot be a definitive proof of the present mechanism because there are other possible alternative explanations in terms of the interaction between halo and bar. For instance, halos might allow bars to become stronger (Athanassoula \& Misiriotis 2002; Athanassoula 2002, 2003). In any case, the present section might be a first step in the search of this effect.
In order to do this, we use first the data from the Mathewson et al. (1992) catalogue on rotation curves, which contains data for 1355 spiral galaxies. We subdivide the sample into 12 groups: morphological type from +3 to +8 (equivalently from $\mathrm{Sb}$ to $\mathrm{Sd}$ ) and each of them with or without bar (either $\mathrm{SB} / \mathrm{SAB}$ or $\mathrm{S} / \mathrm{SA}$; according to the classification in this survey similar to the RC3 catalogue). This is a rough classification, because indeed most of the spiral galaxies should have a bar to some degree (e.g., Knapen et al. 2000; Fathi 2004). From Mathewson et al. (1992), we take the maximum rotation velocity. This catalogue includes data from optical $\mathrm{H}_{\alpha}$ rotation curves and HI profiles. The results are given in Table 1 . The table, although not conclusive, seems to show larger rotation velocities for earlier types (for +4 , the average rotation velocity of barred galaxies is $2.9 \sigma$ higher than the non-barred galaxies) and vice verse for later types (for +7 , the average rotation velocity of barred galaxies is $3.1 \sigma$ lower than the non-barred galaxies). The first thing might be explained in terms of the mechanisms proposed in this paper: throughout their lifetimes, bars help to accrete matter and this is more remarkable in earlier types because the bars are more prominent in these types. The hypothesis that halos allow bars to become stronger is also a good possible explanation; indeed, the simulations in Athanassoula \& Misiriotis (2002) and Athanassoula $(2002,2003)$ refer only to early-type disc galaxies, with no or hardly any gas, and this would explain why this effect is not necessarily seen in later types. The second fact is intriguing: it might have to do with the history of the galaxy, in which these late-type galaxies without bars had one in the past, or with a major role of the spiral arms in non-barred galaxy. Also, errors in the classification of the galaxy type could be the cause of this different velocity in barred and non-barred galaxies (the bar could contribute to wrong visual classification).

HI profiles with extension of the wings up to a $20 \%$ of the maximum trace the halo mass within larger radius, so the width of this HI profile $\left(H W 20 M_{\mathrm{HI}}\right.$ in Table 1) is also considered. The results are similar to the results with the rotation curves for latetype spiral galaxies, and no trend is found for early-type ones.

The same comparison between barred and non-barred galaxies could be useful if made in galaxies in clusters. There are very few galaxies of Mathewson et al. (1992) classified as SB and "in cluster": according to the SIMBAD database (http://simbad.u-strasbg.fr/), only 5 of the 95 barred galaxies are known to be in clusters ("GiC"), so this catalogue is not useful for this purpose. We can use another catalogue with fewer galaxies in total (329 galaxies) but with a higher ratio of cluster members: Vogt et al. (2004). Results are shown in Table 2 for all the galaxies, and in Table 2 only for galaxies in clusters. Nothing new is observed with respect to the Mathewson et al. (1992) analysis, which shows more or less the same trends. Analysis of barred galaxies in clusters (there are in 
Table 2. Comparison of $O W 3\left[\mathrm{~km} \mathrm{~s}^{-1}\right]$ (measured velocity width in optical profiles with corrections for the shape of the rotation curve, cosmological stretch and converted to edge-on viewing measured at twice the scale length), $R W 2\left[\mathrm{~km} \mathrm{~s}^{-1}\right]$ (measured $21 \mathrm{~cm}$ line profile velocity corrected and converted to edge on viewing; Note: average only with the galaxies which have this datum available), $M / L$ [solar units] (corrected mass to light [I-band] ratio; Note: average only with the galaxies which have this datum available) for spiral galaxies in Vogt et al. (2004) catalogue in barred and non-barred cases as a function of the galaxy type. There are no barred galaxies with available data in this catalogue of types +6 or later.

\begin{tabular}{lll}
\hline \hline Galaxy type & Non-barred: $(\mathrm{N}),\langle O W 3\rangle,\langle R W 2\rangle,\langle M / L\rangle$ & Barred: $(\mathrm{N}),\langle O W 3\rangle,\langle R W 2\rangle,\langle M / L\rangle$ \\
\hline+3 & $(94), 353 \pm 12,388 \pm 14,0.47 \pm 0.03$ & $(25), 404 \pm 26,434 \pm 35,0.44 \pm 0.06$ \\
+4 & $(37), 321 \pm 16,359 \pm 21,0.52 \pm 0.04$ & $(3), 419 \pm 36,391 \pm 57,0.76 \pm 0.09$ \\
+5 & $(83), 303 \pm 11,331 \pm 10,0.46 \pm 0.03$ & $(9), 252 \pm 28,295 \pm 43,0.52 \pm 0.11$ \\
\hline
\end{tabular}

Table 3. Same as Table 2 but only with galaxies considered bona fide members of a cluster of galaxies. There is only one barred galaxy of type +4 "in a cluster" of Vogt et al. (2004) catalogue, which is not included.

\begin{tabular}{lll}
\hline \hline Galaxy type & Non-barred: $(\mathrm{N}),\langle O W 3\rangle,\langle R W 2\rangle,\langle M / L\rangle$ & Barred: $(\mathrm{N}),\langle O W 3\rangle,\langle R W 2\rangle,\langle M / L\rangle$ \\
\hline+3 & $(73), 350 \pm 12,393 \pm 15,0.47 \pm 0.03$ & $(19), 384 \pm 31,383 \pm 32,0.40 \pm 0.07$ \\
+5 & $(33), 317 \pm 17,346 \pm 16,0.49 \pm 0.05$ & $(4), 234 \pm 55,321 \pm 91,0.46 \pm 0.19$ \\
\hline
\end{tabular}

total 23 for our analysis; not many, but more than the 5 galaxies in Mathewson et al. 1992) does not show a clearer trend of distinction with the non-barred galaxies: Table 3.

It would be better to obtain information on the total mass of halos rather than the mass within a few tens of $\mathrm{kpc}$, as is the case for rotation curves, but this information is not available for a large sample of galaxies, and the errors in the estimates (through satellites orbit measures; e.g. Conroy et al. 2005) are inaccurate and not available for a large sample of individual galaxies to do statistics.

\section{Conclusions and discussion}

A new mechanism has been proposed in the interaction of bars with the surrounding medium, which produces a variation of energy of the particles crossing the galaxy. On average, the energy gained is equal to the loss energy by these particles, so this does not produce variations of energy in the average halo. However, for the external IGM particles which cross the galaxy, this mechanism causes some of them to be trapped to form part of the halo because their velocities are reduced to less than the escape velocity. By means of this, an extra amount of IGM matter can be accreted onto barred galaxies. The increase in the evaporation of the halo due to bar dynamical friction, however, is negligible.

Rough calculations of the amount of accreted matter show that this must be negligible in most cases with normal expected conditions of density and galaxy-IGM velocity, and only in galaxies of galaxy-IGM velocities $<\approx 70 \mathrm{~km} \mathrm{~s}^{-1}$ with IGM density similar to that of the cluster of galaxies does it produce significant $(>10 \%)$ amounts of halo mass accreted by this mechanism. The IGM density might be lower with motions of the galaxies close to the IGM average motion, or with motion perpendicular to the plane of the galaxy. Clusters of galaxies also have other mechanisms of galaxy stripping that can counteract the accretion; indeed, the IGM and halos are mixed and we could even talk about a common halo for all the galaxies in a cluster. This means that we might find that there is no important increase in mass in the barred galaxies of clusters.

The analysis of observational data of rotation curves shows no clear trends. There is a slight increase in the average mass of early-type barred spiral galaxies with respect to non-barred galaxies of the same type, and viceverse for late-type spiral galaxies, but some sort of selection factor might explain it. No effect could be observed for galaxies embedded in clusters, although we cannot exclude the possibility that the effect proposed in this paper is present to some non-negligible degree because it might be lower than the error bars. In Tables 1-3 the relative error bars of the velocities are higher than $10 \%$, which implies a mass error higher than $20 \%$, so possible slight average variations of less than $20 \%$ in mass would not be detectable.

The fashion in galactic models nowadays is to include very massive halos whose matter comes from the evolution of the initial fluctuations of the large scale structure (e.g., Betancort-Rijo \& López-Corredoira 2002) and the accretion of dwarf galaxies, the hierarchical scenario (e.g., White \& Frenk 1991). The mechanism presented in this paper is part of the possible mechanisms of accretion and it can also be applied to the satellite dwarf galaxies. Nevertheless, if the very massive dark matter halos did not exist and the rotation curves were explained by mechanisms different from dark matter (e.g., MOND, Sanders \& McGaugh 2002; magnetic fields, Battaner \& Florido 2000), the present mechanism could be much more important as a relative contributor to the accretion of matter onto the galaxy, and it would not be detected by analysing rotation curves because these would not be related to the halo mass.

What is noteworthy in this paper is that the present mechanism does not suppose any speculative scenario about the galaxies themselves. The mechanism should be present "always". The only question is whether the amount of accreted matter in barred galaxies is significant enough to be detected or not, because this depends on the conditions of the IGM.

Acknowledgements. Discussions with J. E. Beckman (IAC, Tenerife, Spain) have produced some ideas which were used as a basis for the development of the present paper. Thanks are given to E. Athanassoula (Obs. Marseille, France) and the anonymous referee for helpful comments on the draft of this paper and suggestions to improve it; and T. J. Mahoney (IAC, Tenerife, Spain) for proofreading of this paper. This research has made use of the SIMBAD database, operated at CDS, Strasbourg, France.

\section{References}

Aguerri, J. A. L., Debattista, V. P., \& Corsini, E. M. 2003, MNRAS, 338, 465 Athanassoula, E. 1992, MNRAS, 259, 328

Athanassoula, E. 2002, ApJ, 569, L83

Athanassoula, E. 2003, MNRAS, 341, 1179

Athanassoula, E. 2005, Celest. Mech. Dynam. Astron. 91, 9

Athanassoula, E., \& Misiriotis, A. 2002, MNRAS, 330, 35

Battaglia, G., Helmi, A., Morrison, H., et al. 2005, MNRAS, 364, 433

Battaner, E., \& Florido, E. 2000, Fund. Cosmic Phys., 21, 1

Bell, F. B., McIntosh, D. H., Katz, N., \& Weinberg, M. D. 2003, ApJ, 585, L117

Betancort-Rijo, J., \& López-Corredoira, M. 2002, ApJ, 566, 623

Binney, J., \& Tremaine, S. 1987, Galactic dynamics (Princeton: Princeton University Press) 
Bissantz, N., \& Gerhard, O. E. 2002, MNRAS, 330, 591

Blitz, L., Spergel, D., Teuben, P., Hartmann, D., \& Burton, W. B. 1999, ApJ, 514,818

Braun, R., \& Burton, W. B. 1999, A\&A, 341, 437

Casuso, E., \& Beckman, J. E. 1997, ApJ, 475, 155

Conroy, C., Newman, J. A., Davis, M., et al. 2005, ApJ, 635, 982

Debattista, V. P., \& Sellwood, J. A. 1998, ApJ, 493, L5

Debattista, V. P., \& Sellwood, J. A. 2000, ApJ, 543, 704

Debattista, V. P., Gerhard, O. E., \& Sevenster, M. N. 2002, MNRAS, 334, 355

Dehnen, W., \& McLaughlin, D. E. 2005, MNRAS, 363, 1057

Evans, N. W. 2001, in proceedings of the 3rd International Workshop on the Identification of Dark Matter, ed. N. J. C. Spooner, \& V. Kudryavtsev (Singapore: World Scientific), 85

Fathi, K. 2004, Ph.D. Thesis, Univ. Groningen

Fraternali, F., Binney, J., Oosterloo, T., \& Sancisi, R. 2007, New Astron. Rev., 51,95

Honma, M., \& Sofue, Y. 1996, PASJ, 48, L103

Klypin, A., Kravtsov, A. V., Valenzuela, O., \& Prada, F. 1999, ApJ, 522, 82

Knapen, J. H., Shlosman, I., \& Peletier, R. F. 2000, ApJ, 529, 93

Kuijken, K., \& Gilmore, G. 1989, MNRAS, 239, 605

Kuijken, K., \& Dubinski, J. 1995, MNRAS, 277, 1341

López-Corredoira, M., Beckman, J. E., \& Casuso, E. 1999, A\&A, 351, 920

López-Corredoira, M., Hammersley, P. L., Garzón, F., et al. 2001, A\&A, 373, 139
López-Corredoira, M., Betancort-Rijo, J., \& Beckman, J. E. 2002a, A\&A, 386, 169

López-Corredoira, M., Cabrera-Lavers, A., Garzón, F., \& Hammersley, P. L. 2002b, A\&A, 394, 883

López-Corredoira, M., Cabrera-Lavers, A., Mahoney, T. J., et al. 2007, AJ, 133, 154

Mathewson, D. S., Ford, V. L., \& Buchhorn, M. 1992, ApJS, 81, 413

Moore, B., Ghigna, S., Governato, F., et al. 1999, ApJ, 524, L19

Morris, S. L., \& van den Bergh, S. 1994, ApJ, 427, 696

Pfenninger, D. 1990, A\&A, 230, 55

Rauch, M. 1998, ARA\&A, 36, 267

Rocha-Pinto, H. J., \& Maciel, W. J. 1996, MNRAS, 279, 447

Roussel, H., Sadat, R., \& Blanchard, A. 2000, A\&A, 361, 429

Sandage, A., \& Bedke, J. 1994, The Carnegie Atlas of Galaxies, Carnegie Institution of Washington with The Flintridge Foundation, Washington D.C. Sanders, R. H., \& McGaugh, S. S. 2002, ARA\&A, 40, 263

Sellwood, J. A., \& Debattista, V. P. 2006, ApJ 639, 868

Sellwood, J. A., \& Kosowsky, A. 2001, in Gas \& Galaxy Evolution, ed. J. E. Hibbard, M. P. Rupen, \& J. van Gorkom, ASP, S. Francisco, 311

Sevenster, M. N., Prasenjit, S., Valls-Gabaud, D., \& Fux, R. 1999, MNRAS, 307, 584

Vogt, N. P., Haynes, M. P., Herter, T., \& Giovanelli, R. 2004, AJ, 127, 3273

Weinberg, M. D., \& Katz, N. 2007, MNRAS, 375, 460

White, S., \& Frenk, C. 1991, ApJ, 379, 52 